\title{
Minimización de la penalidad generada por los retrasos en la entrega de proyectos mediante un modelo de programación lineal entera
}

\section{Minimising the penalty generated by project delivery delays using an integer linear programming model

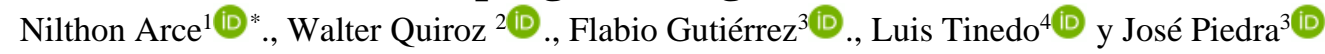

\section{RESUMEN}

La presente investigación tuvo por objetivo minimizar la penalidad total generada por el retraso en la entrega de proyectos operados por una máquina (retroexcavadora). Se empleó el método doble simplex de paso largo con el software GNU Octave (versión 6.2.0). Para estimar la fecha y el orden de entrega de los proyectos antes de la ejecución, se implementó un plan de trabajo para la máquina. La empresa Consultores \& Ejecutores Jhothiza S.R.L. verificó los plazos de entrega ejecutando el modelo y concluyendo que la penalidad óptima fue de S/. 8100.

Palabras clave: Proyecto de desarrollo, modelo matemático, programación lineal, software.

\begin{abstract}
The present research aimed to minimise the total penalty generated by the delay in the delivery of projects operated by a machine (backhoe loader). The double simplex long-pass method was used with the GNU Octave software (version 6.2.0). In order to estimate the date and order of delivery of the projects before execution, a work plan was implemented for the machine. The company Consultores \& Ejecutores Jhothiza S.R.L. verified the delivery times by running the model and concluding that the optimal penalty was S/. 8100.
\end{abstract}

Keywords: Development project, mathematical model, linear programming, software.

DOI: https://doi.org/10.37787/pakamuros-unj.v9i3.222

Recibido: 22/03/2020. Aceptado: 16/07/2021

* Autor para correspondencia

. Universidad Nacional de Jaén, Perú. Email: nilthon_arce@unj.edu.pe

Universidad Nacional de Piura, Perú. Email: wquirozc@posgrado.unp.edu.pe

Universidad Nacional de Piura, Perú. Email: flabio@unp.edu.pe

Universidad Nacional de Jaén, Perú. Email: luis_tinedo@unj.edu.pe

5. Universidad Nacional de Jaén, Perú. Email: jpiedrat@unj.edu.pe 


\section{INTRODUCCIÓN}

Gestionar proyectos de manera adecuada es importante para solucionar problemas de la sociedad, la planificación óptima permite al gerente estimar de manera razonable los recursos, el costo y tiempo del proyecto; evitando así los gastos superfluos durante la ejecución (Sadeq S. et al., 2019). La ley peruana de contrataciones $\mathrm{N}^{\circ} 30225$ indica, el contratista que no cumple con la entrega de la obra en el plazo establecido, tendrá que asumir penalidades que generan sobrecostos (Santos, 2019). En la región Cajamarca, el plazo de ejecución de obras respecto al sugerido en el expediente técnico varió de 70\% a 209\%, esto y más factores generaron variaciones en el costo de 15\% a 90\% (Ángeles, 2017).

La programación lineal entera (PLE) se utiliza como herramienta para resolver distintos problemas relacionados con la investigación de operaciones. Marino et al. (2020), proponen un modelo de PLE para optimizar el tiempo de fabricación de sistemas (Makespan). Gutiérrez et al. (2019), aplican un modelo de PLE para el problema de atraque de barcos en un terminal marítimo de contenedores. Zotelo et al. (2017), proponen un modelo de PLE para minimizar los costes de producción en la elaboración de productos químicos. Bautista et al. (2020), presentan un modelo de PLE con la finalidad de minimizar el coste de almacenamiento de combustible. Duque et al. (2017), aplican un modelo de PLE para minimizar los costes ociosos de una Institución educativa, optimizando los recursos académicos.

Los modelos matemáticos, permite a los gerentes contar con referentes cuantitativos para obtener la solución óptima de los sistemas productivos (Niño, 2003). Este tipo de programación es fundamental para solucionar problemas de organizaciones en las que se tiene que tomar importantes decisiones con la finalidad de optimizar los resultados (Escobar et al., 2012).

Los problemas de secuenciación de tareas permiten alcanzar objetivos como por ejemplo minimizar el tiempo total transcurrido, minimizar el retraso total, minimizar el retraso máximo, minimizar el costo de producción, y más; logrando un objetivo se puede obtener otros por estar estrechamente relacionados (Ballesteros et al., 2006). El modelo planteado se aplica en organizaciones que se dedican a vender y ejecutar proyectos (empresas de consultoría o de ingeniería), también en compañías que trabajan en proyectos de innovación (Acuña et al., 2013).

El retraso en la entrega de proyectos es un problema frecuente y común en las empresas, ya que generan penalidades muy costosas que afectan el patrimonio de las mismas. Por esto, en la investigación se implementó un modelo de PLE para minimizar la penalidad total generada por el retraso en la entrega de proyectos ejecutados por una máquina. Estos modelos de optimización contribuyen a disminuir diversos tipos de costes, ahorrando recursos que generan un crecimiento económico estable de las organizaciones. 
En base a lo mencionado el objetivo fue minimizar la penalidad total generada por el retraso en la entrega de tres proyectos ejecutados por la empresa Consultores \& Ejecutores Jhothiza S.R.L.

\section{MATERIALES Y MÉTODOS}

Modelo matemático general para minimizar la penalidad dada por el retraso en la entrega de " $n$ " proyectos operados por una máquina

Figura 1, muestra la red de precedencia de operaciones en la ejecución de “n” proyectos. Disponiendo de una máquina retroexcavadora, donde se genera la pregunta ¿cómo determinar un plan de trabajo que permita minimizar la multa total generada por el retraso en la entrega de los proyectos?; este plan investiga la fecha de inicio de todas las operaciones a ejecutarse.

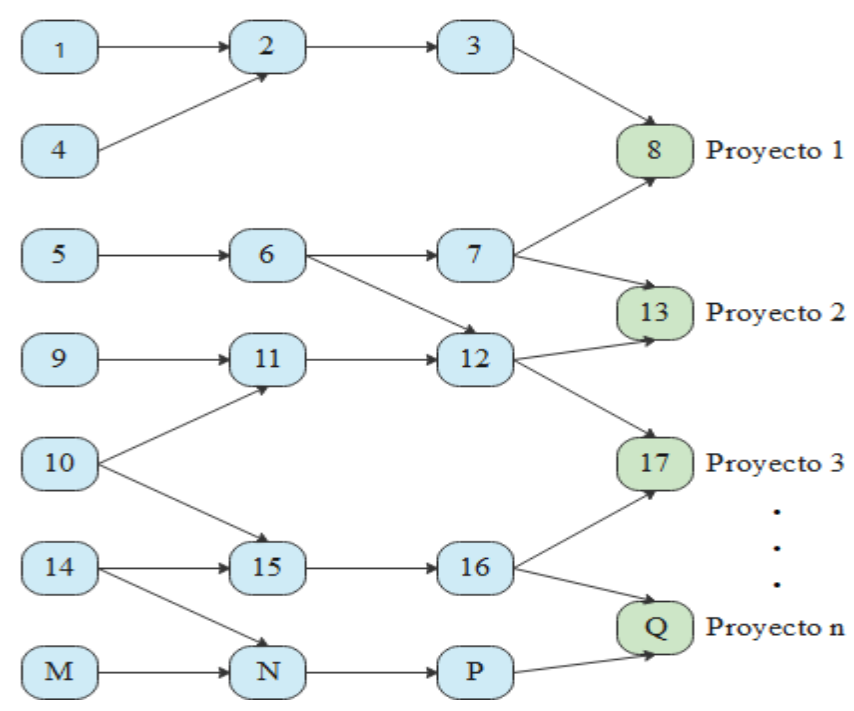

Figura 1. Red de precedencia de operaciones en la ejecución de "n" proyectos

Taha (2012), estudia modelos matemáticos que minimizan multas, en los que las restricciones son de no interferencia, de precedencia y de tiempo de entrega.

\section{Restricciones de no interferencia}

La Figura 2, garantiza que no se procesen dos operaciones al mismo tiempo, siendo:

$x_{i}$ : Fecha de inicio de la operación $i$

$x_{j}$ : Fecha de inicio de la operación $j$

$t_{i}$ : Tiempo de duración de la operación $i$ 


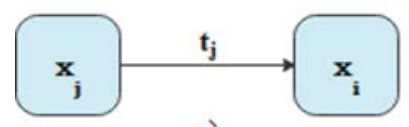

a) $\mathbf{0}$

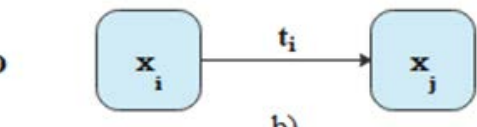

Figura 2. No interferencia de operaciones.

La Figura 2-a), muestra que la operación $j$ se realiza antes que la operación $i$, mientras que la Figura 2b) muestra lo contrario, esto permite obtener las relaciones:

$$
x_{i}-x_{j} \geq t_{j} \quad \vee \quad x_{j}-x_{i} \geq t_{i}
$$

De (1), según Medina et al. (2008), para determinar la restricción activa dejando la otra como no activa, se define la variable binaria auxiliar $y_{i j}$, como sigue:

$$
y_{i j}=\left\{\begin{array}{lll}
1, & \text { si } i \text { antes que } j \\
0, & \text { si } j \text { antes que } i
\end{array}\right.
$$

Incluyendo en (1), (2) y $M$ que es un número suficientemente grande para las condiciones del problema, las restricciones de no interferencia son las siguientes:

$$
x_{i}-x_{j} \geq t_{j}-M y_{i j} \wedge x_{j}-x_{i} \geq t_{i}-M\left(1-y_{i j}\right)
$$

\section{Restricciones de precedencia}

La Figura 3, muestra que para iniciar la operación $j$ primero se realizará la operación $i$, es decir, estas son precedentes directos.

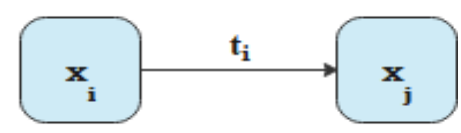

Figura 3. Operaciones precedentes directas.

De la Figura 3, las restricciones de precedencia son las siguientes:

$$
x_{j}-x_{i} \geq t_{i}
$$

\section{Restricciones de tiempo de entrega}

Para determinar estas restricciones, se considera:

$x_{u}$ : Fecha de inicio de la última operación $u$ del proyecto $P$

$t_{u}$ : Tiempo de duración de la operación $u$

$f_{P}$ : Fecha límite de entrega del proyecto $P$

$v_{P}$ : Variable de desviación

$m_{P}$ : Multa por día de retraso en la entrega del proyecto $P$ 


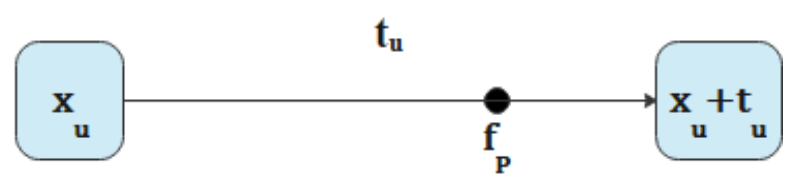

Figura 4. Entrega del proyecto después de la fecha límite

De la Figura 4, se tiene:

$$
x_{u}+t_{u}>f_{P}
$$

de (5) se obtiene

$$
x_{u}+t_{u}+v_{P}=f_{P}
$$

De (6), la variable de desviación $v_{P} \leq 0$, en este caso se genera una penalidad por entregar el proyecto después de la fecha límite.

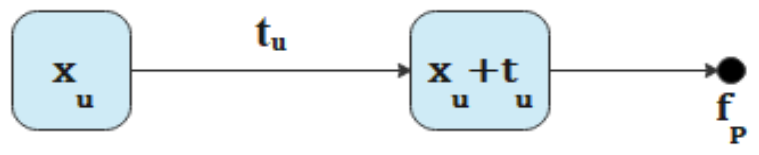

Figura 5. Entrega del proyecto antes de la fecha límite.

De la Figura 5, se tiene:

$$
x_{u}+t_{u}<f_{P}
$$

de (7) se obtiene

$$
x_{u}+t_{u}+v_{P}=f_{P}
$$

De (8), la variable de desviación $v_{P} \geq 0$, en este caso el proyecto se entrega antes de la fecha límite, es decir, no hay penalización.

Sustituyendo la variable de desviación $v_{P}$ por:

$$
v_{P}=s_{P a}-s_{P b} ; s_{P a}, s_{P b} \geq 0
$$

Cuando $s_{P b}>0$ y $s_{P a}=0$, de la ecuación (9) la variable $v_{P}<0$, esto genera una multa por día de retraso en la entrega del proyecto (Figura 4); pero si $s_{P a}>0$ y $s_{P b}=0$, la variable $v_{P}>0$, lo que indica que no hay multa en la entrega del proyecto (Figura 5).

Remplazando (9) en (8), las restricciones de tiempo de entrega son las siguientes:

$$
x_{u}+t_{u}+s_{P a}-s_{P b}=f_{P}
$$

El modelo tiene por objetivo determinar un plan de trabajo para la máquina que permita minimizar la multa total generada por la variable de desviación $s_{P b}$. Por lo tanto, la función objetivo está dada por: 


$$
\min Z=\sum_{P=1}^{n} m_{P} \cdot s_{P b}
$$

Finalmente, el modelo matemático general para obtener la penalidad mínima óptima se expresa como sigue:

$\min Z=\sum_{P=1}^{n} m_{P} \cdot s_{P b}$

Sujeta a las restricciones:

$x_{i}-x_{j} \geq t_{j}-M y_{i j}$

$x_{j}-x_{i} \geq t_{i}-M\left(1-y_{i j}\right)$

$x_{j}-x_{i} \geq t_{i}$

$x_{u}+t_{u}+s_{P a}-s_{P b}=f_{P}$

donde:

$x_{i}, s_{P a}, s_{P b} \in Z_{0}^{+}$

$y_{i j} \in\{0,1\}$

M es un número suficientemente grande

\section{Caso de estudio}

La empresa Consultores \& Ejecutores Jhothiza S.R.L. con sede principal en la provincia de Jaén, tiene un plan de trabajo para ejecutar tres proyectos al mismo tiempo utilizando una máquina (Retroexcavadora). Debido al cobro de penalidades elevadas por retraso de entregas anteriores, se diseñó junto al gerente la red de precedencia de operaciones para ejecutar los tres proyectos (Figura 6).

Para ello se consideró las variables; tiempo que utiliza la máquina para ejecutar cada operación (Tabla 1), fecha límite de entrega por proyecto y penalidad por día de retraso (Tabla 2) 


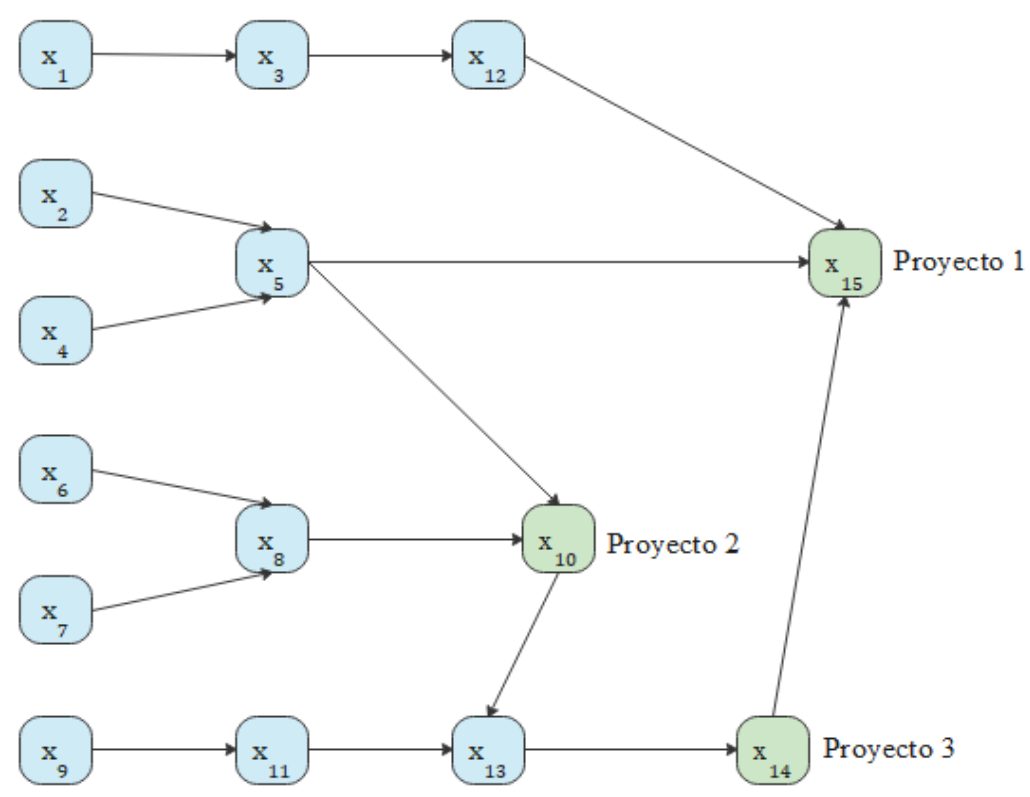

Figura 6. Precedencia de operaciones en la ejecución de tres proyectos.

Tabla 1. Tiempo que utiliza la máquina para ejecutar cada operación.

\begin{tabular}{cc}
\hline Operación & $\begin{array}{c}\text { Tiempo de ejecución } \\
\text { (días) }-\left(\boldsymbol{t}_{\boldsymbol{i}}\right)\end{array}$ \\
\hline 1 & 15 \\
2 & 25 \\
3 & 37 \\
4 & 10 \\
5 & 24 \\
6 & 20 \\
7 & 22 \\
8 & 18 \\
9 & 13 \\
10 & 35 \\
11 & 26 \\
12 & 21 \\
13 & 31 \\
14 & 29 \\
15 & 28 \\
\hline
\end{tabular}


Tabla 2. Fecha límite de entrega por proyecto y penalidad por día de retraso.

\begin{tabular}{ccc}
\hline Proyectos & $\begin{array}{c}\text { Fecha límite de entrega } \\
(\text { días })-\left(\boldsymbol{f}_{\boldsymbol{P}}\right)\end{array}$ & $\begin{array}{c}\text { Penalidad } \\
\text { (soles/día) }-\left(\boldsymbol{m}_{\boldsymbol{P}}\right)\end{array}$ \\
\hline$P_{1}$ & 344 & 500 \\
$P_{2}$ & 146 & 200 \\
$P_{3}$ & 248 & 300 \\
\hline
\end{tabular}

\section{Modelación matemática del caso de estudio}

Para la modelación del caso de estudio, se consideró:

$x_{i}$ : Fecha de inicio de la operación $i ; i=1,2,3, \ldots, 15$

$S_{P a}$ : Variable de desviación que no genera multa en la entrega del proyecto $P, P=1,2$ y 3

$s_{P b}$ : Variable de desviación que si genera multa en la entrega del proyecto $P, P=1,2$ y 3

$y_{i j}$ : Variable binaria

$M=2000$ es un número suficientemente grande para las condiciones dadas

Aplicando (12), el modelo matemático respecto al caso de estudio, se representa de la manera:

$$
\min Z=500 s_{1 b}+200 s_{2 b}+300 s_{3 b}
$$

(13) está sujeta a restricciones de no interferencia, de precedencia y de tiempo de entrega:

Tabla 3. Restricciones de no interferencia, en total 116

\begin{tabular}{|c|c|c|c|}
\hline \multicolumn{4}{|c|}{ Restricciones de No Interferencia } \\
\hline 1. $\mathrm{x}_{1}-\mathrm{x}_{2}+2000 \mathrm{y}_{12} \geq 25$ & 30. $-\mathrm{x}_{2}+\mathrm{x}_{7}-2000 \mathrm{y}_{27} \geq-1975$ & 59. $\mathrm{x}_{4}-\mathrm{x}_{6}+2000 \mathrm{y}_{46} \geq 20$ & 88. $-\mathrm{x}_{6}+\mathrm{x}_{11}-2000 \mathrm{y}_{611} \geq-1980$ \\
\hline 2. $-\mathrm{x}_{1}+\mathrm{x}_{2}-2000 \mathrm{y}_{12} \geq-1985$ & 31. $\mathrm{x}_{2}-\mathrm{x}_{8}+2000 \mathrm{y}_{28} \geq 18$ & 60. $-\mathrm{x}_{4}+\mathrm{x}_{6}-2000 \mathrm{y}_{46} \geq-1990$ & 89. $\mathrm{x}_{6}-\mathrm{x}_{12}+2000 \mathrm{y}_{612} \geq 21$ \\
\hline 3. $\mathrm{x}_{1}-\mathrm{x}_{4}+2000 \mathrm{y}_{14} \geq 10$ & 32. $-\mathrm{x}_{2}+\mathrm{x}_{8}-2000 \mathrm{y}_{28} \geq-1975$ & 61. $\mathrm{x}_{4}-\mathrm{x}_{7}+2000 \mathrm{y}_{47} \geq 22$ & 90. $-\mathrm{x}_{6}+\mathrm{x}_{12}-2000 \mathrm{y}_{612} \geq-1980$ \\
\hline 4. $-\mathrm{x}_{1}+\mathrm{x}_{4}-2000 \mathrm{y}_{14} \geq-1985$ & 33. $\mathrm{x}_{2}-\mathrm{x}_{9}+2000 \mathrm{y}_{29} \geq 13$ & 62. $-\mathrm{x}_{4}+\mathrm{x}_{7}-2000 \mathrm{y}_{47} \geq-1990$ & 91. $\mathrm{x}_{7}-\mathrm{x}_{9}+2000 \mathrm{y}_{79} \geq 13$ \\
\hline 5. $\mathrm{x}_{1}-\mathrm{x}_{5}+2000 \mathrm{y}_{15} \geq 24$ & 34. $-\mathrm{x}_{2}+\mathrm{x}_{9}-2000 \mathrm{y}_{29} \geq-1975$ & 63. $\mathrm{x}_{4}-\mathrm{x}_{8}+2000 \mathrm{y}_{48} \geq 18$ & 92. $-\mathrm{x}_{7}+\mathrm{x}_{9}-2000 \mathrm{y}_{79} \geq-1978$ \\
\hline 6. $-\mathrm{x}_{1}+\mathrm{x}_{5}-2000 \mathrm{y}_{15} \geq-1985$ & 35. $\mathrm{x}_{2}-\mathrm{x}_{11}+2000 \mathrm{y}_{211} \geq 26$ & 64. $-\mathrm{x}_{4}+\mathrm{x}_{8}-2000 \mathrm{y}_{48} \geq-1990$ & 93. $\mathrm{x}_{7}-\mathrm{x}_{11}+2000 \mathrm{y}_{711} \geq 26$ \\
\hline 7. $x_{1}-x_{6}+2000 y_{16} \geq 20$ & 36. $-\mathrm{x}_{2}+\mathrm{x}_{11}-2000 \mathrm{y}_{211} \geq-1975$ & 65. $\mathrm{x}_{4}-\mathrm{X}_{9}+2000 \mathrm{y}_{49} \geq 13$ & 94. $-\mathrm{x}_{7}+\mathrm{x}_{11}-2000 \mathrm{y}_{711} \geq-1978$ \\
\hline 8. $-\mathrm{x}_{1}+\mathrm{x}_{6}-2000 \mathrm{y}_{16} \geq-1985$ & 37. $\mathrm{x}_{2}-\mathrm{x}_{12}+2000 \mathrm{y}_{212} \geq 21$ & 66. $-\mathrm{x}_{4}+\mathrm{x}_{9}-2000 \mathrm{y}_{49} \geq-1990$ & 95. $\mathrm{x}_{7}-\mathrm{x}_{12}+2000 \mathrm{y}_{712} \geq 21$ \\
\hline 9. $\mathrm{x}_{1}-\mathrm{x}_{7}+2000 \mathrm{y}_{17} \geq 22$ & 38. $-\mathrm{x}_{2}+\mathrm{x}_{12}-2000 \mathrm{y}_{212} \geq-1975$ & 67. $\mathrm{x}_{4}-\mathrm{x}_{11}+2000 \mathrm{y}_{411} \geq 26$ & 96. $-\mathrm{x}_{7}+\mathrm{x}_{12}-2000 \mathrm{y}_{712} \geq-1978$ \\
\hline 10. $-\mathrm{x}_{1}+\mathrm{x}_{7}-2000 \mathrm{y}_{17} \geq-1985$ & 39. $\mathrm{x}_{3}-\mathrm{x}_{4}+2000 \mathrm{y}_{34} \geq 10$ & 68. $-\mathrm{x}_{4}+\mathrm{x}_{11}-2000 \mathrm{y}_{411} \geq-1990$ & 97. $\mathrm{x}_{8}-\mathrm{x}_{9}+2000 \mathrm{y}_{89} \geq 13$ \\
\hline 11. $\mathrm{x}_{1}-\mathrm{x}_{8}+2000 \mathrm{y}_{18} \geq 18$ & 40. $-\mathrm{x}_{3}+\mathrm{x}_{4}-2000 \mathrm{y}_{34} \geq-1963$ & 69. $\mathrm{x}_{4}-\mathrm{x}_{12}+2000 \mathrm{y}_{412} \geq 21$ & 98. $-\mathrm{x}_{8}+\mathrm{x}_{9}-2000 \mathrm{y}_{89} \geq-1982$ \\
\hline 12. $-\mathrm{x}_{1}+\mathrm{x}_{8}-2000 \mathrm{y}_{18} \geq-1985$ & 41. $\mathrm{x}_{3}-\mathrm{x}_{5}+2000 \mathrm{y}_{35} \geq 24$ & 70. $-\mathrm{x}_{4}+\mathrm{x}_{12}-2000 \mathrm{y}_{412} \geq-1990$ & 99. $\mathrm{x}_{8}-\mathrm{x}_{11}+2000 \mathrm{y}_{811} \geq 26$ \\
\hline 13. $\mathrm{x}_{1}-\mathrm{x}_{9}+2000 \mathrm{y}_{19} \geq 13$ & 42. $-\mathrm{x}_{3}+\mathrm{x}_{5}-2000 \mathrm{y}_{35} \geq-1963$ & 71. $x_{5}-x_{6}+2000 y_{56} \geq 20$ & 100. $-\mathrm{x}_{8}+\mathrm{x}_{11}-2000 \mathrm{y}_{811} \geq-1982$ \\
\hline 14. $-\mathrm{x}_{1}+\mathrm{x}_{9}-2000 \mathrm{y}_{19} \geq-1985$ & 43. $\mathrm{x}_{3}-\mathrm{x}_{6}+2000 \mathrm{y}_{36} \geq 20$ & 72. $-\mathrm{x}_{5}+\mathrm{x}_{6}-2000 \mathrm{y}_{56} \geq-1976$ & 101. $\mathrm{x}_{8}-\mathrm{x}_{12}+2000 \mathrm{y}_{812} \geq 21$ \\
\hline 15. $\mathrm{x}_{1}-\mathrm{x}_{10}+2000 \mathrm{y}_{110} \geq 35$ & 44. $-\mathrm{x}_{3}+\mathrm{x}_{6}-2000 \mathrm{y}_{36} \geq-1963$ & 73. $x_{5}-x_{7}+2000 y_{57} \geq 22$ & 102. $-\mathrm{x}_{8}+\mathrm{x}_{12}-2000 \mathrm{y}_{812} \geq-1982$ \\
\hline
\end{tabular}




\begin{tabular}{|c|c|c|c|}
\hline 16. $-\mathrm{x}_{1}+\mathrm{x}_{10}-2000 \mathrm{y}_{110} \geq-1985$ & 45. $\mathrm{x}_{3}-\mathrm{x}_{7}+2000 \mathrm{y}_{37} \geq 22$ & 74. $-\mathrm{x}_{5}+\mathrm{x}_{7}-2000 \mathrm{y}_{57} \geq-1976$ & 103. $\mathrm{x}_{9}-\mathrm{X}_{10}+2000 \mathrm{y}_{910} \geq 35$ \\
\hline 17. $x_{1}-x_{11}+2000 y_{111} \geq 26$ & 46. $-\mathrm{x}_{3}+\mathrm{x}_{7}-2000 \mathrm{y}_{37} \geq-1963$ & 75. $\mathrm{x}_{5}-\mathrm{x}_{8}+2000 \mathrm{y}_{58} \geq 18$ & 104. $-\mathrm{x}_{9}+\mathrm{x}_{10}-2000 \mathrm{y}_{910} \geq-1987$ \\
\hline 18. $-\mathrm{x}_{1}+\mathrm{x}_{11}-2000 \mathrm{y}_{111} \geq-1985$ & 47. $\mathrm{x}_{3}-\mathrm{x}_{8}+2000 \mathrm{y}_{38} \geq 18$ & 76. $-\mathrm{x}_{5}+\mathrm{x}_{8}-2000 \mathrm{y}_{58} \geq-1976$ & 105. $\mathrm{x}_{9}-\mathrm{X}_{12}+2000 \mathrm{y}_{912} \geq 21$ \\
\hline 19. $\mathrm{x}_{1}-\mathrm{x}_{13}+2000 \mathrm{y}_{113} \geq 31$ & 48. $-\mathrm{x}_{3}+\mathrm{x}_{8}-2000 \mathrm{y}_{38} \geq-1963$ & 77. $\mathrm{x}_{5}-\mathrm{x}_{9}+2000 \mathrm{y}_{59} \geq 13$ & 106. $-\mathrm{x}_{9}+\mathrm{x}_{12}-2000 \mathrm{y}_{912} \geq-1987$ \\
\hline 20. $-\mathrm{x}_{1}+\mathrm{x}_{13}-2000 \mathrm{y}_{113} \geq-1985$ & 49. $\mathrm{x}_{3}-\mathrm{x}_{9}+2000 \mathrm{y}_{39} \geq 13$ & 78. $-\mathrm{x}_{5}+\mathrm{x}_{9}-2000 \mathrm{y}_{59} \geq-1976$ & 107. $\mathrm{x}_{10}-\mathrm{x}_{11}+2000 \mathrm{y}_{1011} \geq 26$ \\
\hline 21. $x_{1}-x_{14}+2000 y_{114} \geq 29$ & 50. $-\mathrm{x}_{3}+\mathrm{x}_{9}-2000 \mathrm{y}_{39} \geq-1963$ & 79. $\mathrm{x}_{5}-\mathrm{x}_{11}+2000 \mathrm{y}_{511} \geq 26$ & 108. $-\mathrm{x}_{10}+\mathrm{x}_{11}-2000 \mathrm{y}_{1011} \geq-1965$ \\
\hline 22. $-\mathrm{x}_{1}+\mathrm{x}_{14}-2000 \mathrm{y}_{114} \geq-1985$ & 51. $\mathrm{x}_{3}-\mathrm{x}_{10}+2000 \mathrm{y}_{310} \geq 35$ & 80. $-\mathrm{x}_{5}+\mathrm{x}_{11}-2000 \mathrm{y}_{511} \geq-1976$ & 109. $\mathrm{x}_{10}-\mathrm{x}_{12}+2000 \mathrm{y}_{1012} \geq 21$ \\
\hline 23. $\mathrm{x}_{2}-\mathrm{x}_{3}+2000 \mathrm{y}_{23} \geq 37$ & 52. $-\mathrm{x}_{3}+\mathrm{x}_{10}-2000 \mathrm{y}_{310} \geq-1963$ & 81. $\mathrm{x}_{5}-\mathrm{x}_{12}+2000 \mathrm{y}_{512} \geq 21$ & 110. $-\mathrm{x}_{10}+\mathrm{x}_{12}-2000 \mathrm{y}_{1012} \geq-1965$ \\
\hline 24. $-\mathrm{x}_{2}+\mathrm{x}_{3}-2000 \mathrm{y}_{23} \geq-1975$ & 53. $\mathrm{x}_{3}-\mathrm{x}_{11}+2000 \mathrm{y}_{311} \geq 26$ & 82. $-\mathrm{x}_{5}+\mathrm{x}_{12}-2000 \mathrm{y}_{512} \geq-1976$ & 111. $\mathrm{x}_{11}-\mathrm{x}_{12}+2000 \mathrm{y}_{1112} \geq 21$ \\
\hline 25. $\mathrm{x}_{2}-\mathrm{x}_{4}+2000 \mathrm{y}_{24} \geq 10$ & 54. $-\mathrm{x}_{3}+\mathrm{x}_{11}-2000 \mathrm{y}_{311} \geq-1963$ & 83. $\mathrm{x}_{6}-\mathrm{x}_{7}+2000 \mathrm{y}_{67} \geq 22$ & 112. $-\mathrm{x}_{11}+\mathrm{x}_{12}-2000 \mathrm{y}_{1112} \geq-1974$ \\
\hline 26. $-\mathrm{x}_{2}+\mathrm{x}_{4}-2000 \mathrm{y}_{24} \geq-1975$ & 55. $\mathrm{x}_{3}-\mathrm{x}_{13}+2000 \mathrm{y}_{313} \geq 31$ & 84. $-\mathrm{x}_{6}+\mathrm{x}_{7}-2000 \mathrm{y}_{67} \geq-1980$ & 113. $\mathrm{x}_{12}-\mathrm{x}_{13}+2000 \mathrm{y}_{1213} \geq 31$ \\
\hline 27. $\mathrm{x}_{2}-\mathrm{x}_{6}+2000 \mathrm{y}_{26} \geq 20$ & 56. $-\mathrm{x}_{3}+\mathrm{x}_{13}-2000 \mathrm{y}_{313} \geq-1963$ & 85. $\mathrm{x}_{6}-\mathrm{x}_{9}+2000 \mathrm{y}_{69} \geq 13$ & 114. $-\mathrm{x}_{12}+\mathrm{x}_{13}-2000 \mathrm{y}_{1213} \geq-1979$ \\
\hline 28. $-\mathrm{x}_{2}+\mathrm{x}_{6}-2000 \mathrm{y}_{26} \geq-1975$ & 57. $\mathrm{x}_{3}-\mathrm{x}_{14}+2000 \mathrm{y}_{314} \geq 29$ & 86. $-\mathrm{x}_{6}+\mathrm{x}_{9}-2000 \mathrm{y}_{69} \geq-1980$ & 115. $\mathrm{x}_{12}-\mathrm{x}_{14}+2000 \mathrm{y}_{1214} \geq 29$ \\
\hline 29. $\mathrm{x}_{2}-\mathrm{x}_{7}+2000 \mathrm{y}_{27} \geq 22$ & 58. $-\mathrm{x}_{3}+\mathrm{x}_{14}-2000 \mathrm{y}_{314} \geq-1963$ & 87. $x_{6}-x_{11}+2000 y_{611} \geq 26$ & 116. $-\mathrm{x}_{12}+\mathrm{x}_{14}-2000 \mathrm{y}_{1214} \geq-1979$ \\
\hline
\end{tabular}

Tabla 4. Restricciones de precedencia, en total quince

\begin{tabular}{c}
\hline Restricciones de Precedencia \\
\hline 1. $-\mathrm{x}_{1}+\mathrm{x}_{3} \geq 15$ \\
2. $-\mathrm{x}_{2}+\mathrm{x}_{5} \geq 25$ \\
3. $-\mathrm{x}_{4}+\mathrm{x}_{5} \geq 10$ \\
4. $-\mathrm{x}_{6}+\mathrm{x}_{8} \geq 20$ \\
5. $-\mathrm{x}_{7}+\mathrm{x}_{8} \geq 22$ \\
6. $-\mathrm{x}_{5}+\mathrm{x}_{10} \geq 24$ \\
7. $-\mathrm{x}_{8}+\mathrm{x}_{10} \geq 18$ \\
8. $-\mathrm{x}_{9}+\mathrm{x}_{11} \geq 13$ \\
9. $-\mathrm{x}_{3}+\mathrm{x}_{12} \geq 37$ \\
10. $-\mathrm{x}_{10}+\mathrm{x}_{13} \geq 35$ \\
11. $-\mathrm{x}_{11}+\mathrm{x}_{13} \geq 26$ \\
12. $-\mathrm{x}_{13}+\mathrm{x}_{14} \geq 31$ \\
13. $-\mathrm{x}_{5}+\mathrm{x}_{15} \geq 24$ \\
14. $-\mathrm{x}_{12}+\mathrm{x}_{15} \geq 21$ \\
15. $-\mathrm{x}_{14}+\mathrm{x}_{15} \geq 29$ \\
\hline
\end{tabular}


Tabla 5. Restricciones de tiempo de entrega, en total tres.

\begin{tabular}{c}
\hline Restricciones de Tiempo de Entrega \\
\hline $\begin{array}{l}\text { 1. } \mathrm{x}_{15}+\mathrm{s}_{1 \mathrm{a}}-\mathrm{S}_{1 \mathrm{~b}}=316 \\
\text { 2. } \mathrm{x}_{10}+\mathrm{s}_{2 \mathrm{a}}-\mathrm{S}_{2 \mathrm{~b}}=111 \\
\text { 3. } \mathrm{x}_{14}+\mathrm{s}_{3 \mathrm{a}}-\mathrm{S}_{3 \mathrm{~b}}=219\end{array}$ \\
\hline
\end{tabular}

donde:

$x_{i}, s_{P a}, s_{P b} \in Z_{0}^{+}$

El modelo se implementó en el software GNU Octave, versión 6.2.0; ejecutándose en una computadora personal equipada con procesador Intel (R) Core (TM) i5-8250U CPU @ 1.60 GHz 1.80 GHz con 6.00 GB RAM. Para la solución del caso de estudio, el software empleó el método doble simplex de paso largo.

\section{RESULTADOS}

Figura 7, se observa la fecha de inicio de las quince operaciones a ejecutarse para entregar los tres proyectos.

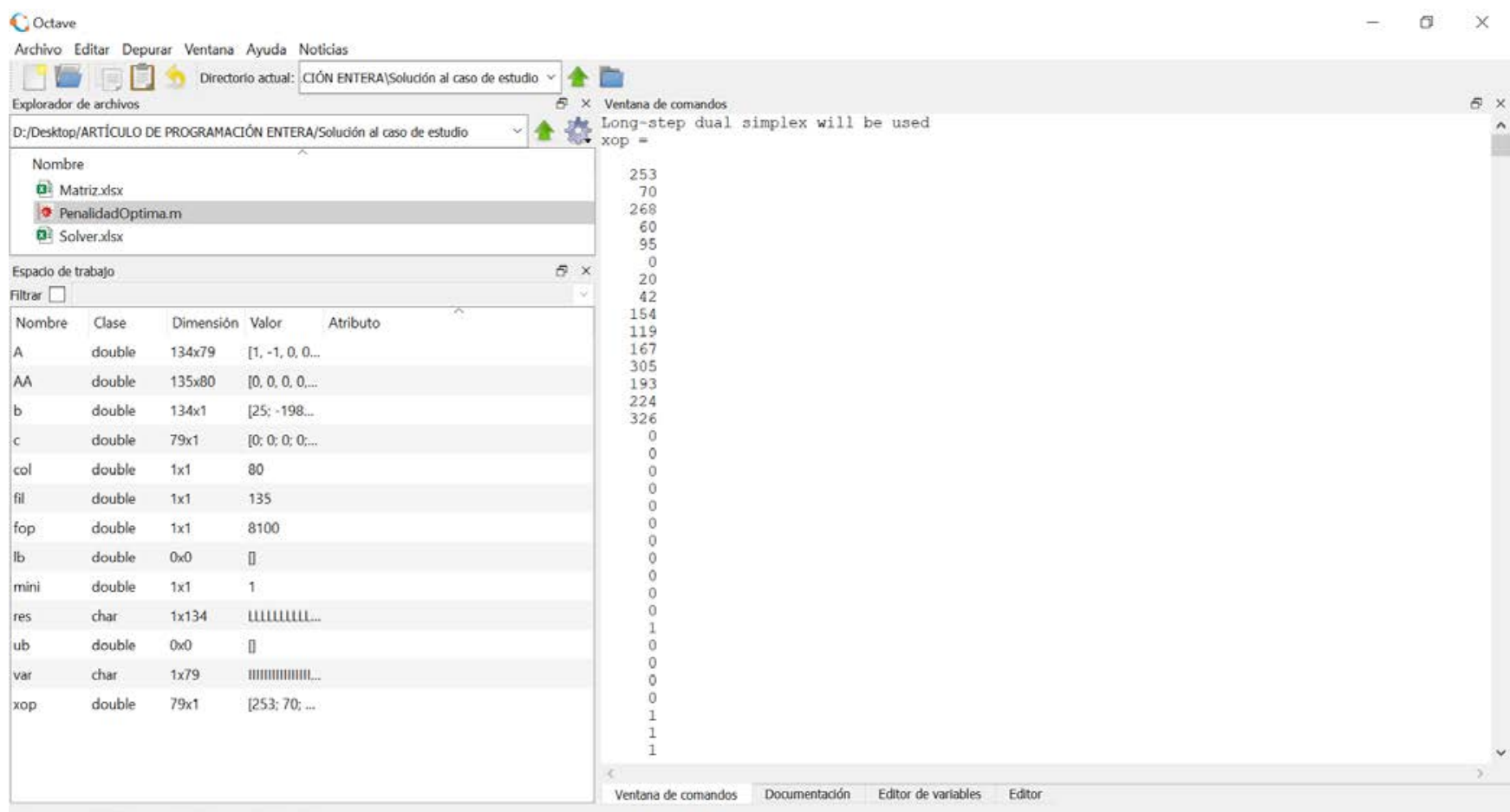

Figura 7. Fecha de inicio de las operaciones ejecutadas por la máquina 
Figura 8, se observa la solución del modelo matemático respecto al caso de estudio; el Proyecto 1, Proyecto 2 y Proyecto 3 se entregaron con 10, 8 y 5 días de retraso respectivamente, además la penalidad óptima sería S/. 8100.

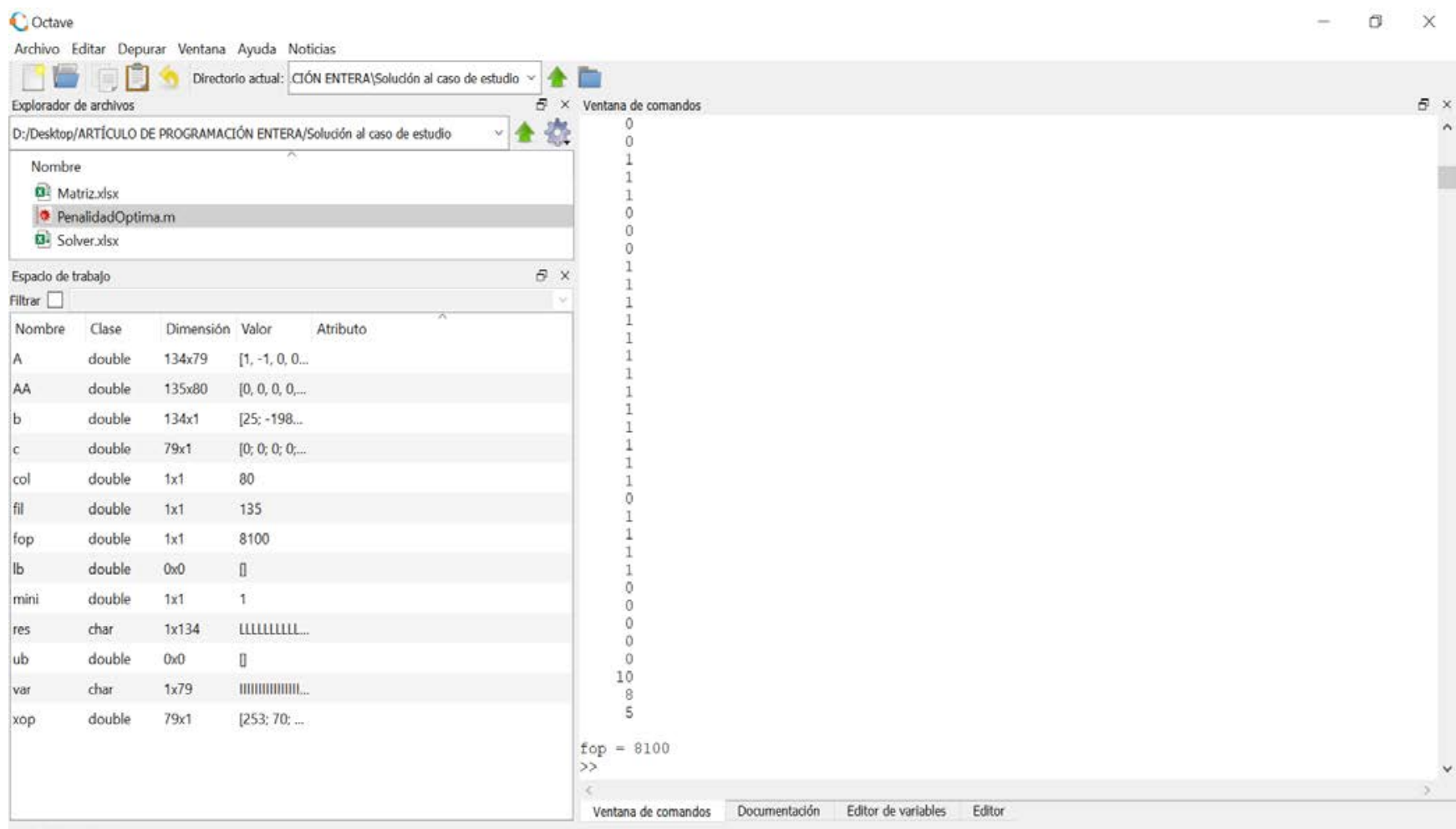

Figura 8. Solución del modelo matemático respecto al caso de estudio

En la Tabla 6, se verifica el plan de trabajo implementado para la máquina que permitió minimizar la multa total generada por el retraso en la entrega de los tres proyectos.

Tabla 6. Plan de trabajo que minimizó la multa total generada por el retraso en la entrega de los Proyectos

\begin{tabular}{cccccc}
\hline $\begin{array}{c}\text { Plan de } \\
\text { Trabajo }\end{array}$ & $\begin{array}{c}\text { Fecha de Inicio } \\
\left(\boldsymbol{x}_{\boldsymbol{i}}\right)\end{array}$ & $\begin{array}{c}\text { Tiempo de Ejecución } \\
\left(\boldsymbol{t}_{\boldsymbol{i}}\right)\end{array}$ & $\begin{array}{c}\text { Fecha Límite de Entrega } \\
\left(\boldsymbol{f}_{\boldsymbol{P}}\right)\end{array}$ & $\begin{array}{c}\text { Retraso } \\
\left(\boldsymbol{s}_{\boldsymbol{P} \boldsymbol{b}}\right)\end{array}$ & $\begin{array}{c}\text { Multa } \\
\left(\boldsymbol{m}_{\boldsymbol{P}}\right)\end{array}$ \\
\hline 6 & 0 & 20 & & & \\
7 & 20 & 22 & & & \\
8 & 42 & 18 & & & \\
4 & 60 & 10 & & & \\
2 & 70 & 25 & & & \\
5 & 95 & 24 & & & \\
10 & 119 & 35 & & & \\
9 & 154 & 13 & & & \\
11 & 167 & 26 & & & \\
13 & 193 & 31 & & & \\
\hline
\end{tabular}




\begin{tabular}{cccccc}
\hline 14 & 224 & 29 & 248 & 5 & S/. 300 \\
1 & 253 & 15 & & & \\
3 & 268 & 37 & & & \\
12 & 305 & 21 & 344 & 10 & S/. 500 \\
15 & 326 & 28 & & \\
\hline
\end{tabular}

\section{DISCUSIÓN}

Los modelos de optimización basados en programación lineal entera, permiten tomar decisiones que disminuyen favorablemente los gastos en la empresa (Bermúdez, 2011). En una planta textil se programó trabajos con fechas límite de entrega, la función objetivo del modelo propuesto fue minimizar el tiempo total de procesamiento para cumplir con las entregas en los plazos pactados, la solución del modelo respecto al caso de estudio se orientó a obtener el mínimo óptimo del tiempo de fabricación por producto, esto permitió cumplir de manera ordenada con las fechas de entrega reduciendo las penalidades (Arango et al., 2013). En los resultados obtenidos, se verifica que el plan de trabajo implementado para la máquina permitió a la empresa Consultores \& Ejecutores Jhothiza S.R.L. saber la fecha y el orden de entrega de los tres proyectos ejecutados.

La programación de la producción en las organizaciones depende de su configuración productiva o del número de máquinas, se programó un flowshop flexible (FFS) logrando minimizar la tardanza total que permitió alcanzar la penalidad óptima (Salazar \& Sarzuri, 2015). El modelo propuesto generó un mejor desempeño de la empresa en estudio, ya que logró minimizar el retraso total de entrega de trabajos, reduciendo la penalidad por incumplimiento (Ortiz \& Ruiz, 2017). El modelo aplicado a la empresa Consultores \& Ejecutores Jhothiza S.R.L. también logró minimizar el retraso total de entrega de los proyectos, alcanzando la penalidad óptima mediante el plan de trabajo implementado para la máquina.

En la investigación que realizaron Escobar et al. (2020), obtuvieron como resultado que el modelo respecto al caso de estudio es favorable para la disminución de sus pasivos. El modelo de programación para minimizar el makepan y el consumo de energía, demostró que la estrategia para minimizar el costo total es promover el consumo responsable manteniendo la producción, los resultados indican que esta estrategia reduce los costos de producción, mejora el uso de recursos y promueve el consumo responsable (Moreira et al., 2019). En la presente investigación, se obtuvo como resultado que el modelo respecto al caso de estudio es favorable para la disminución de los días de retraso en la entrega de los tres proyectos. 
El modelo propuesto proveyó mejores soluciones en la disminución de las órdenes atrasadas, incrementando el nivel de servicio al cliente en la organización, es importante que para mejorar su desempeño la empresa cuente con un sistema de programación de producción (Riquelme, 2020). Los modelos de optimización contribuyen a disminuir diversos tipos de costes, esto permite ahorrar recursos que generan un crecimiento económico estable de las empresas (Bermúdez, 2011). Por los resultados obtenidos en la presente investigación, la empresa donde se aplicó el modelo matemático cuenta con un plan de trabajo óptimo para la única Retroexcavadora que actualmente dispone; esta deberá realizar quince operaciones que están en el cronograma de los tres proyectos ejecutados al mismo tiempo.

\section{CONCLUSIONES}

Se logró minimizar la penalidad total generada por el retraso de entrega de los tres proyectos, es decir, la penalidad óptima fue S/. 8100.

Para aceptar la ejecución de los tres proyectos, la empresa Consultores \& Ejecutores Jhothiza S.R.L. verificó los plazos de entrega ejecutando el modelo.

En un trabajo futuro, se pretende ampliar el modelo para un número mayor de máquinas y proyectos, programar el modelo en herramientas de optimización que permitan indizar las variables para tener un código corto, optimizado y rápido; así mismo obtener modelos que contemplen la incertidumbre que puede darse en algunas variables como el retraso en el inicio de las tareas o en su tiempo de realización.

\section{REFERENCIAS BIBLIOGRÁFICAS}

Acuña, S. Y., Madiedo, E., \& Ortiz, N. R. (2013). Modelo de programación lineal binaria para el balance de carga de trabajo en el problema de asignación de proyectos. Ingeniería y Universidad, 17(1), 167-181.

Ángeles, C. H. (2017). Costo y plazo de obras de saneamiento, ejecutadas por la unidad ejecutora de programas regionales de Cajamarca, periodo del 2010 al 2016. Universidad Nacional de Cajamarca.

Arango, J. A., Giraldo, J. A., \& Castrillón, O. D. (2013). Programación de Máquinas Paralelas no Relacionadas con Tiempos de Montaje dependientes de la Secuencia y Entrada Dinámica usando Algoritmos Genéticos. Información Tecnológica, 24(3), 73-84. 
Ballesteros, P. P., Ballesteros, D. P., \& Bernal, M. E. (2006). Programación disyuntiva para n tareas en m máquinas en producción por pedidos. Scientia et Technica, 1(30), 273-278.

Bautista, J., Batet, L., \& Mateo, M. (2020). Minimización del coste de almacenaje de combustible nuclear gastado en cápsulas MPC-32 con programación lineal entera mixta: Caso de carga en una etapa.

Bermúdez, Y. (2011). Aplicaciones de programación lineal, entera y mixta. Ingeniería Industrial. Actualidad y Nuevas Tendencias, 2(7), 85-104.

Duque, E. A., Gonzalez, J. D., \& Ramírez, J. D. (2017). Diseño de un modelo de programación lineal para la asignación de aulas en la Institución Universitaria Esumer, Colombia. Revista ESPACIOS, 38(10). http://www.revistaespacios.com/a17v38n10/17381003.html

Escobar, D. F., Garcés, J. A., \& Restrepo, J. H. (2012). Aplicación de la programación entera binaria para resolver el problema simple de balanceo de línea de ensamble: Un caso de estudio. Scientia et Technica, 2(50), 85-90.

Escobar, J. W., Marceles, J. J., \& Quevedo, D. F. (2020). Modelo matemático para la programación de la producción en compañías fabricantes de alambres y cables para la construcción. Revista ESPACIOS, 41(24).

Gutiérrez, F., Lujan, E., Asmat, R., \& Vergara, E. (2019). Fuzziness in the berth allocation problem. Recent Advances in Computational Optimization. Springer, Cham., 149-174.

Marino, E. D., Su, R., \& Basile, F. (2020). Makespan optimization using Timed Petri Nets and Mixed Integer Linear Programming Problem. IFAC-PapersOnLine, 53(4), 129-135. https://doi.org/10.1016/j.ifacol.2021.04.073

Medina, P. D., Cruz, E. A., \& Restrepo, J. H. (2008). Programación de trabajos en una maquina utilizando un modelo de programación lineal entera. Scientia et Technica, 3(40), 111-116.

Moreira, J. C. E., Perdigão, L., Gadelha, F., \& Freitas, M. J. (2019). Uma formulação de programação matemática para minimizar o makespan e o custo de energia em um problema de sequenciamento em máquinas paralelas. 2. https://proceedings.science/p/106875?lang=pt-br

Niño, M. L. (2003). Modelamiento a través de la programación lineal entera mixta del problema de programación de operaciones en el taller de trabajo. Revista UIS Ingenierías, 2(1), 9-17.

Ortiz, S. E., \& Ruiz, C. R. (2017). Modelo de Programación Lineal Entera Mixta para la Programación de Sistemas tipo Job-Shop Flexible en Entornos Make to Order. INGE CUC, 13(2), 28-34. https://doi.org/10.17981/ingecuc.13.2.2017.03 
Riquelme, M. C. (2020). Programación de una máquina considerando variados lotes para múltiples productos y tiempos de setup dependientes de la secuencia. http://repositorio.udec.cl/jspui/handle/11594/6272

Sadeq S., G., Hassan, S., García, R., Pérez, I., Madera, J., \& Piñero, P. (2019). Algoritmo de estimación de distribución con tratamiento de restricciones en el modelo probabilístico en problemas de scheduling. Investigación Operacional, 40(5), 674-686.

Salazar, E., \& Sarzuri, R. A. (2015). Algoritmo genético mejorado para la minimización de la tardanza total en un flowshop flexible con tiempos de preparación dependientes de la secuencia. Ingeniare. Revista chilena de ingeniería, 23(1), 118-127.

Santos, C. (2019). Factores que inciden en retraso de proyectos públicos en la etapa de ejecución en la cuidad de Cerro de Pasco. Universidad Nacional Daniel Alcides Carrión.

Taha, H. A. (2012). Investigación de operaciones (Novena). Pearson Educación.

Zotelo, Y. R., Mula, J., Díaz-Madroñero, M., \& González, E. G. (2017). Plan maestro de producción basado en programación lineal entera para una empresa de productos químicos. Revista de Métodos Cuantitativos para la Economía y la Empresa, 24, 147-168. 